\title{
The Arabidopsis WRR4A and WRR4B paralogous NLR proteins both confer recognition of multiple Albugo candida effectors
}

Amey Redkar ${ }^{1,2^{*}}$, Volkan Cevik ${ }^{1,3^{*}}$, Kate Bailey ${ }^{1}$, Oliver J. Furzer ${ }^{1,4}$, Sebastian Fairhead

${ }^{1,5}$, M. Hossein Borhan ${ }^{6}$, Eric B. Holub ${ }^{5}$ and Jonathan D.G. Jones ${ }^{1 \#}$

1. The Sainsbury Laboratory, University of East Anglia, Norwich NR4 7UH, United Kingdom

2. Department of Genetics, University of Córdoba, Córdoba 14071, Spain

10 3. The Milner Centre for Evolution, Department of Biology and Biochemistry, University of Bath, Bath BA2 7AY, United Kingdom

4. Department of Biology, University of North Carolina, Chapel Hill, NC 27599, USA

5. University of Warwick, School of Life Sciences, Warwick Crop Centre, Wellesbourne, CV35 9EF, United Kingdom

15 6. Agriculture and Agri-Food Canada, 107 Science Place, Saskatoon, SK. S7N 0X2, Canada

* These authors contributed equally

${ }^{\#}$ Corresponding author:

20 Jonathan D.G. Jones, Email: jonathan.jones@tsl.ac.uk

\section{Statistics}

Introduction: 1,226

Materials and Methods: 1,323

25 Results: 2,536

Discussion: 1,073

Total: 6,158 words

No. of figures: 5 (all in colour)

Supporting Information: Fig. S1-S9. Table S1-S3. 


\section{Summary}

35 The oomycete Albugo candida causes white blister rust, an important disease of Brassica crops. Distinct races of $A$. candida are defined by their specificity for infecting different host species.

The White Rust Resistance 4 (WRR4) locus in Col-0 accession of Arabidopsis thaliana contains three genes that encode TIR-NLR resistance proteins. The Col-0 alleles of $W R R 4 A$ and WRR $4 B$ confer resistance to at least four $A$. candida races $(2,7$ and 9 from B. juncea, B. rapa and B. oleracea, respectively, and Race 4 from Capsella bursapastoris). Resistance mediated by both paralogs can be overcome by Col-0-virulent isolates of Race 4.

45

After comparing repertoires of candidate effectors in resisted and resistance-breaking strains, we used transient co-expression in tobacco or Arabidopsis to identify effectors recognized by $W R R 4 A$ and $W R R 4 B$. A library of CCG effectors from four $A$. candida races was screened for $W R R 4 A$ - or $W R R 4 B$ - dependent elicitation of hypersensitive response (HR). These CCG genes were validated for WRR-dependent HR by bombardment assays in wild type Col-0, wrr $4 A$ or wrr $4 B$ mutants.

Our analysis revealed eight WRR4A-recognized CCGs and four WRR4B-recognized CCGs. Remarkably, the N-terminal region of 100 amino acids after the secretion signal is sufficient for $W R R 4 A$ recognition of these eight recognized effectors. This multiple recognition capacity potentially explains the broad-spectrum resistance to many $A$. candida races conferred by WRR 4 paralogs.

\section{Keywords}

65 Albugo candida, Effectors, Oomycete, hypersensitive response, TIR-NLR, WRR4. 


\section{Introduction}

Co-evolution of hosts and their parasites remains a fascinating biological problem. Most plant pathogens fail to colonize most potential hosts, in part because of defense responses initiated upon pathogen perception by cell surface or intracellular immune receptors (Jones \& Dangl, 2006). Successful pathogens evade host recognition and suppress defense via effectors (Toruño et al., 2016). In co-evolving systems, genetic variation in resistance and susceptibility to particular pathogen races is usually specified by loci encoding Nucleotide Binding, Leucine-rich Repeat (NLR) intracellular immune receptors (Tomborski \& Krasileva, 2020). Plant NLRs are under diversifying selection compared to the rest of the genome (Monteiro \& Nishimura, 2018). NLR genes are classified as TIR-NLR (TNL) with N-terminal Toll/Interleukin-1/Resistance domains, CC-NLR (CNL) with a coiled-coil (CC) domain, or RPW8-NLR (RNL) with Resistance to Powdery mildeW8 (RPW8) domains (Meyers et al. 2003; Zhang et al. 2016).

80 Variation in NLR repertoires has been investigated by Resistance gene enrichment sequencing (RenSeq) (Jupe et al., 2013). NLR immune receptor polymorphism and diversity in 64 accessions of Arabidopsis thaliana has been defined using RenSeq (Van de Weyer et al., 2019).

NLR-mediated effector-triggered immunity (ETI) often leads to a hypersensitive

85 cell death response (HR) upon effector recognition. Recent work using estradiolinducible AvrRps4 activation of ETI in the absence of pattern triggered immunity (PTI), revealed mutual potentiation between PTI and ETI to confer resistance (Ngou et al., 2021). Effectors can be recognized via direct interaction with an NLR protein, described as the 'ligand- receptor model' (Jia et al., 2000; Dodds et al., 2006). The flax (Linum usitatissimum) L6 NLR protein directly recognizes variants of the flax rust fungus (Melampsora lini) effector AvrL567 (Ravensdale et al., 2012). Alternatively, some NLRs can detect multiple sequence-unrelated effectors indirectly. Such NLRs either "guard" host proteins that are targeted by multiple effectors (guardee model) (Dangl \& Jones, 2001), or guard "decoy" proteins that have evolved to mimic the host target (van der

95 Hoorn \& Kamoun, 2008). Effector recognition by some NLRs involves a post-LRR (PL) domain. Recent structural investigations in two TNLs, RPP1 and Roq1 revealed a Cterminal jelly-roll and Ig-like domain (C-JID) in mediating effector binding to form a tetrameric resistosome upon activation (Ma et al., 2020; Martin et al., 2020).

White blister rust in a wide range of crop and wild Brassica species is caused by oomycete pathogens in the genus Albugo (Holub et al., 1995; Voglmayr \& Riethmüller, 
2006; Choi et al., 2007). The disease symptoms of white pustules resemble sporulation of basidiomycete rust fungi. Dispersal is via dehydrated sporangiospores (Heller \& Thines, 2009). Jouet et al. (2019) verified that different phylogenetic races of $A$. candida exhibit distinct host specificities for infecting different crop and wild species of

105 Brassicaceae. Important examples include Races 2, 7 and 9 from major crop species (B. juncea, B. rapa and B. oleracea, respectively) and Race 4 from the common weed Capsella bursa-pastoria. Genome comparisons revealed ancient genetic exchange and introgression amongst these $A$. candida races (McMullan et al., 2015). A. candida species have a remarkable capacity to suppress host resistance to other infections (Cooper et al., 110 2008; Belhaj et al., 2017; Prince et al., 2017) which creates an immune-compromised state in the colonized host enabling growth of non-adapted pathogens.

White Rust Resistance 4 (WRR4) from A. thaliana confers resistance to A. candida (Borhan et al., 2008). The locus contains three paralogs in A. thaliana accession Columbia (Col-0) that encode TNLs. The Col-0 allele of WRR4A confers resistance to four $A$.

115 candida races in A. thaliana (Borhan et al., 2008), and also as a transgene to Ac2 V in Brassica crops (Borhan et. al, 2010; Cevik et al., 2019). The Col-0 allele of WRR4B also confers resistance to Brassica-infecting A. candida races Ac2V and AcBoT (Cevik et al., 2019). Although resistance in Col-0 appears to be broad spectrum, WRR4 resistancebreaking strains (e.g., AcEx1) have been collected from white rust pustules on floral

120 stems of wild $A$. thaliana or A. halleri. These are natural pathotypic variants of $A$. candida Race 4 (Fairhead, 2016; Jouet et al., 2019). Resistance to this Race 4 pathotype occurs in A. thaliana, such as in the accession Oystese (Oy-0) which also maps to the WRR4 locus (Fairhead, 2016; Castel et al., 2021).

A. thaliana accession Wassilewskija (Ws-2) is resistant in leaves to A. candida 125 Race 2 and 7. The WRR4 locus in Ws-2 is disrupted compared to Col-0 by deletions or sequence variation of WRR4 paralogs. Ws-2 contains two divergent paralogs (Van de Weyer et al., 2019) and one of these (a Ws-2 allele of WRR4B) confers resistance to isolates of $A$. candida Race 2 (from $B$. juncea). Both Col-0 and Ws-2 alleles of WRR $4 B$ also confer resistance in transgenic Brassicas (Cevik et al., 2019). Conceivably, allelic variation of TNL paralogs at the WRR4 locus provides multiple genes that could control white rust in major Brassica crops. Stacking multiple WRR genes should promote durability. Thus, identifying and understanding function of $A$. candida effectors recognized by alleles of $W R R 4 A$ and $W R R 4 B$ would help choose the most effective transgene combinations to use for Albugo control in Brassica crops. 

Phytophthora, Pythium and downy mildews) are translocated into host cells (Kamoun, 2006; Haas et al., 2009). These effectors typically carry an N-terminal signal peptide for secretion and an RxLR motif that is implicated in host translocation (Whisson et al., 2007; Dou et al., 2008). However, in the P. infestans effector Avr3a, the RxLR motif is cleaved 140 during secretion, and how it promotes translocation still remains unclear (Wawra et al., 2017). RxLR effectors show enhanced polymorphism and positive selection for diversification towards their C termini (Rehmany et al., 2005; Win et al., 2007).

Genome analysis of two Albugo species (Albugo laibachii and A. candida) revealed a new class of oomycete effector-like proteins that carry a "CHxC" motif 145 (Kemen et al., 2011). Albugo lacks RxLR-encoding proteins compared to the Peronosporales. Re-sequencing of an $A$. candida race from B. juncea (Ac2V) using long reads revealed massive expansion of effector-like proteins with a $\mathrm{CHxC}$-like motif, which was reclassified as $\mathrm{CX}_{2} \mathrm{CX}_{5} \mathrm{G}$ and abbreviated to $\mathrm{CCG}$, resembling previously identified CHxC proteins in A. laibachii (Kemen et al., 2011; Furzer et al., 2021). CCG proteins

150 show no homology to other oomycete secreted proteins. Their high sequence divergence resembles that of RxLR effectors (Furzer et al., 2021), and supports their investigation as candidates for being the effectors recognized by White Rust Resistance (WRR) genes.

In this study, we compared the genomes of resisted and resistance-breaking strains of $A$. candida to define candidate differentially-recognized CCG effectors. We screened 155 a library of CCG secreted proteins, mainly from $A$. candida races 2 and 4, to identify potential effectors recognized by Col-0 alleles of WRR $4 A$ and WRR $4 B$. Agrobacteriummediated transient co-expression was used in a pre-screen to identify pairwise combinations of effector and $R$-allele that activated an HR. Twelve CCG candidates were identified including eight recognized by Col-WRR4A and four by Col-WRR4B. Positive

160 candidates were then validated for HR recognition by a bombardment assay in Col-0 wild type and mutants (wrr4A or wrr4B) of A. thaliana. Several of these CCGs are absent or else show expression polymorphism in the Col-0 virulent isolate AcEx1. To further characterize this WRR4 recognition, we focused on WRR4A-recognized CCGs, for which the N-terminal region is sufficient for recognition. Our data reveal a novel capacity for recognition of multiple effectors of $A$. candida by two distinct WRR 4 paralogs.

\section{MATERIALS AND METHODS}

\section{Plant material and growth conditions}


Wild type and mutant $A$. thaliana accessions used in this study included Col-0, 170 Wassilewskija-2 (Ws-2), Col-0_wrr4a-6 (Borhan et al., 2008), Col-0_wrr4b (Cevik et al., 2019) and the recombinant inbred line (RIL) CW20 that was derived from a cross between Col-5 x Ws-2 and in this RIL, WRR4 locus is the only known WRR locus introgressed from Col-5 (Fairhead, 2016). Seeds were sown directly on compost and were grown at $21^{\circ} \mathrm{C}$, with 10 hours of light and 14 hours of dark, at $75 \%$ humidity. For $N$.

175 tabacum and $N$. benthamiana, plants were grown on compost at $21^{\circ} \mathrm{C}$, with cycles of 16 hours of light and 8 hours of dark, at 55\% humidity.

\section{A. candida infection assay}

For leaf inoculations, zoospores harvested from previous leaf infections, were suspended

180 in water $\left(\sim 10^{5}\right.$ spores $\left./ \mathrm{ml}\right)$ and incubated on ice for $30 \mathrm{~min}$ for releasing of the zoospores from sporangia due to cold shock. The spore suspension was then sprayed on plants using a Humbrol spray gun (Hornby Hobbies Ltd, Sandwich, UK) with a volume equal to $~ 700$ $\mu 1 /$ plant and plants were incubated at $4^{\circ} \mathrm{C}$ in the dark overnight for efficient zoospore germination. Infected plants were kept under 10-hour light $\left(21^{\circ} \mathrm{C}\right)$ and 14-hour dark

$185\left(16^{\circ} \mathrm{C}\right)$ cycles. Phenotypes were monitored between 7 to 10 days post inoculation (dpi) and macroscopic symptoms were readily visible during this period.

The sequential infection assay was done as described previously in McMullan et al., 2015. We developed $A$. candida race specific PCR primers by comparing the genome sequences for regions that were unique to the tested $A$. candida races AcNc2 and AcEx1. (Table S1).

190 Primers were designed to amplify these regions from genomic DNA extracted from each isolate. Primary inoculum was sprayed onto control and test plants. In the case of AcEx1 WRR $4 A$ mediated defense suppression assays, both $A$. thaliana Ws-2 and CW-20 were inoculated. The inoculated plants were incubated in the dark at $4^{\circ} \mathrm{C}$ overnight. Same number of plants treated with water served as mock control. At 7 dpi a secondary infection

195 with the avirulent $A$. candida race AcNc2 was performed on $50 \%$ of the plants while the remaining 50\% were again mock inoculated with water. The co-inoculated plants were returned to the growth cabinet and incubated for a further 8 dpi. Tissue was harvested and washed in sterile water to remove surface adhering spores, and flash frozen in liquid N2. DNA was prepared using a DNeasy Plant Mini Kit (Qiagen, CA, USA) as described in manufacturer's instructions. PCR was performed using race-specific primers and products were visualized on a $1 \%$ agarose gel. 


\section{Gene cloning and plasmid construction}

Cloning of genes were carried out using Uracil-Specific Excision Reagent (USER) 205 method (Geu-Flores et al., 2007). Genes with 5' and 3' regulatory sequences were cloned into LBJJ233-OD vector (containing a FAST-Red selectable marker) pre-linearized with PacI and Nt.BbvcI. For overexpression, plant genes and CCG effectors, which lack introns, were cloned into pre-linearized LBJJ234-OD (containing a FAST-Red selectable marker, CaMV 35S promoter and Ocs terminator) or pICH86988 (containing Kan

210 selectable marker, CaMV 35S promoter and Ocs terminator). Genes were C-terminally tagged either with a His-FLAG (HF tag) or a yellow fluorescent protein (YFP) tag. Briefly, the candidate CCG effector was PCR amplified from one of the $A$. candida races $(\mathrm{AcNc} 2, \mathrm{AcEm} 2$ or $\mathrm{Ac} 2 \mathrm{~V})$ for the high throughput screen for $W R R 4 A$ - recognized CCGs and from race Ac2V for WRR4B- recognized CCGs. The genomic DNA was used as a

215 template with KAPA HiFi Uracil+ enzyme, following the manufacturer's protocol. To obtain mutant versions of CCG28 $8^{\mathrm{AAs} 28-130}$-YFP carrying mutations in the CCG motif (CCG exchanged to AAG, CAA, CAG and AAA), site directed mutagenesis was performed with QuikChange Multi Site-Directed Mutagenesis Kit (Stratagene, Santa Clara, USA) following manufacturer's instructions. A list of primers and vectors are

220 indicated in Table S1. WRR4A and WRR $4 B$ under $35 \mathrm{~S}$ promoter were cloned in pICH86988 from the Col-0 genomic DNA. All the plasmids were transformed in to Escherichia coli DH10B electro-competent cells selected with appropriate antibiotics and purified using a Qiaprep Spin Miniprep Kit (Qiagen). Positive clones were transformed in Agrobacterium tumefaciens strain GV3101 and used in infiltrations for transient expression experiments.

\section{Transient expression in $N$. tabacum or $N$. benthamiana leaves and cell death assay}

A. tumefaciens strains were streaked on selective media and incubated at $28{ }^{\circ} \mathrm{C}$ for 24 hours. The streaked inoculum was transferred to liquid LB medium with appropriate

230 antibiotic and incubated at $28^{\circ} \mathrm{C}$ for 24 hours in a shaking incubator at 200 rotations $/ \mathrm{min}$ (rpm). The resulting cultures was centrifuged at 3,000 rpm for $5 \mathrm{~min}$ and resuspended in infiltration buffer (10 $\mathrm{mM} \mathrm{MgCl}_{2}, 10 \mathrm{mM}$ MES, $150 \mu \mathrm{M}$ acetosyringone $\left.\mathrm{pH} 5.6\right)$ at $\mathrm{OD}_{600}$ of $0.4\left(2 \times 10^{8} \mathrm{cfu} / \mathrm{ml}\right)$. For co-expression, each bacterial suspension was adjusted to $\mathrm{OD}_{600}$ of 0.4. The abaxial surface of 4-weeks old N. tabacum or 5 weeks old N. benthamiana

235 were infiltrated with $1 \mathrm{ml}$ needleless syringe. Cell death was phenotyped two to four days after infiltration. For the $W R R 4 B$ recognition assay, macroscopic cell death phenotypes 
were scored according to the HR index modified from (Segretin et al., 2014) ranging from 0 (no visible necrosis) to 6 (full necrosis).

\section{Particle bombardment in $\boldsymbol{A}$. thaliana and luciferase assay}

Transient protein expression in Arabidopsis leaves was performed by biolistic gene transfer. $1.0 \mu \mathrm{m}$ Tungsten particles (Bio-Rad) were coated with the plasmids coding for the indicated CCG genes driven under CaMV 35S promoter (Table S1). Bombardment was performed using a PDS-1000/He system (Bio-Rad) onto 4-weeks-old Arabidopsis

245 leaves. After bombardment the leaves were incubated in small vials with the leaf petiole immersed in water, for 48 hours post bombardment (hpb). The leaves were then frozen in liquid $\mathrm{N} 2$ and stored at $-80{ }^{\circ} \mathrm{C}$ until further processing.

For the luciferase assay a Dual Reporter Luciferase Assay system (Promega) was used. Four transiently bombarded leaf events were pooled together and crushed in lysis

250 buffer. The extract was centrifuged at $12,000 \mathrm{rpm}$ for $10 \mathrm{~min}$ at $4^{\circ} \mathrm{C} .20 \mu \mathrm{l}$ of the lysate was then dispersed in 96 well plate in triplicates and analyzed on Varioskan Flash Instrument by injecting $100 \mu \mathrm{l}$ of luciferase assay reagent II, which includes substrate and reaction buffer. A 10 second read time was used to measure luciferase activity for each well.

\section{Gene expression measurement by RT-qPCR}

For gene expression analysis, RNA was isolated from three biological replicates and used for reverse transcription quantitative PCR (RT-qPCR) after cDNA synthesis. Briefly, RNA was extracted using the RNeasy Plant Mini Kit (Qiagen) with the DNase treatment

260 (Qiagen). Reverse transcription was carried out using the SuperScript IV Reverse Transcriptase (ThermoFisher). RT-qPCR was performed using CFX96 Touch Real-Time PCR (Bio-Rad). Primers for qPCR analysis of different CCGs are enlisted in Table $\mathbf{S 1 .}$ Data were analysed using the double $\Delta \Delta C$ T method (Livak \& Schmittgen, 2001) by calculating the relative expression of candidate $\mathrm{CCG}$ in relation to the A. candida EF1a as a house keeping reference gene.

\section{Protein extraction and Western Blot}

Protein was extracted from Agrobacterium infiltrated N. benthamiana leaves at 72 hpi as previously described (Sarris et al., 2015). Briefly, leaves were harvested and ground in 270 liquid N2, and extracted in GTEN buffer (10\% glycerol, $100 \mathrm{mM}$ Tris-HCl, $\mathrm{pH} 7.5,1$ 
mM EDTA, $150 \mathrm{mM} \mathrm{NaCl}, 5 \mathrm{mM}$ 1,4-dithiothreitol (DTT), $1 \times$ Complete protease inhibitor mixture (Roche) and 0.2\% (V/V) Noniodet P-40). $30 \mu$ of the supernatant from the sample extract was used to elute the samples by boiling in loading buffer. For SDSPAGE, samples were heated for $10 \mathrm{~min}$ at $95{ }^{\circ} \mathrm{C}$ for denaturation. After electrophoresis, separated proteins were transferred to PVDF (Merck) membranes for immunoblotting. Membranes were blocked for 2 hours in 5\% nonfat milk, probed with horseradish peroxidase (HRP)-conjugated antibodies for overnight. Chemiluminescence detection for proteins was carried out by incubating the membrane with developing reagents (SuperSignal West Pico \& West Femto), using ImageQuant LAS 4000 (Life Sciences, USA).

\section{Statistical analysis}

Statistical analysis was carried out using the GraphPad Prism 9.0 software (San Diego, USA). The statistical test employed is provided in the figure legends.

\section{RESULTS}

The $W R R 4 A^{\mathrm{Col}-0}$ and $W R R 4 B^{\mathrm{Col}-0 / \mathrm{Ws}-2}$ provide broad spectrum resistance to $A$. candida races

$W R R 4 A^{\text {Col-0 }}$ confers full green resistance (GR) to multiple $A$. candida races (Cevik et al., 290 2019; Borhan et al., 2008) including the Race 2 isolate Ac2V and two highly similar Race 4 isolates AcNc2 and AcEm2 (McMullan et al., 2015) (Fig. 1 and Table S2). The Arabidopsis accession Ws-2 which lacks WRR4A is fully susceptible to AcEm2 but Col0-wrr4-6 mutant shows necrotic resistance (NR) rather than GR as observed with wildtype (WT) Col-0, due to the presence of additional WRR genes in Col-0. This suggests $W R R 4 A^{\text {Col-o }}$ contributes to full GR to AcEm2 (Fig. 1). A Race 4 isolate AcEx1 overcomes $W R R 4 A^{\mathrm{Col}-0}$ mediated resistance but triggers chlorosis in infected adult Col-0 plants (Fig. 1). The Col-0wrr4-6 mutant exhibits full susceptibility to AcEx1 indicating that $W R R 4 A^{\mathrm{Col}-0}$ confers partial resistance to AcEx1.

The $W R R 4 B^{\mathrm{Col}-0 / \mathrm{Ws}-2}$ paralogs also confer resistance to Ac2V in A. thaliana and 300 in transgenic B. juncea (Cevik et al., 2019). However, Ws-2, which lacks WRR4A, exhibits full GR phenotype in adult leaves following inoculation with either Ac2V or Ac7V indicating that $W R R 4 B^{\mathrm{Ws}-2}$ also confers adult plant resistance to Races 2 and 7 of A. candida (Table S2). 

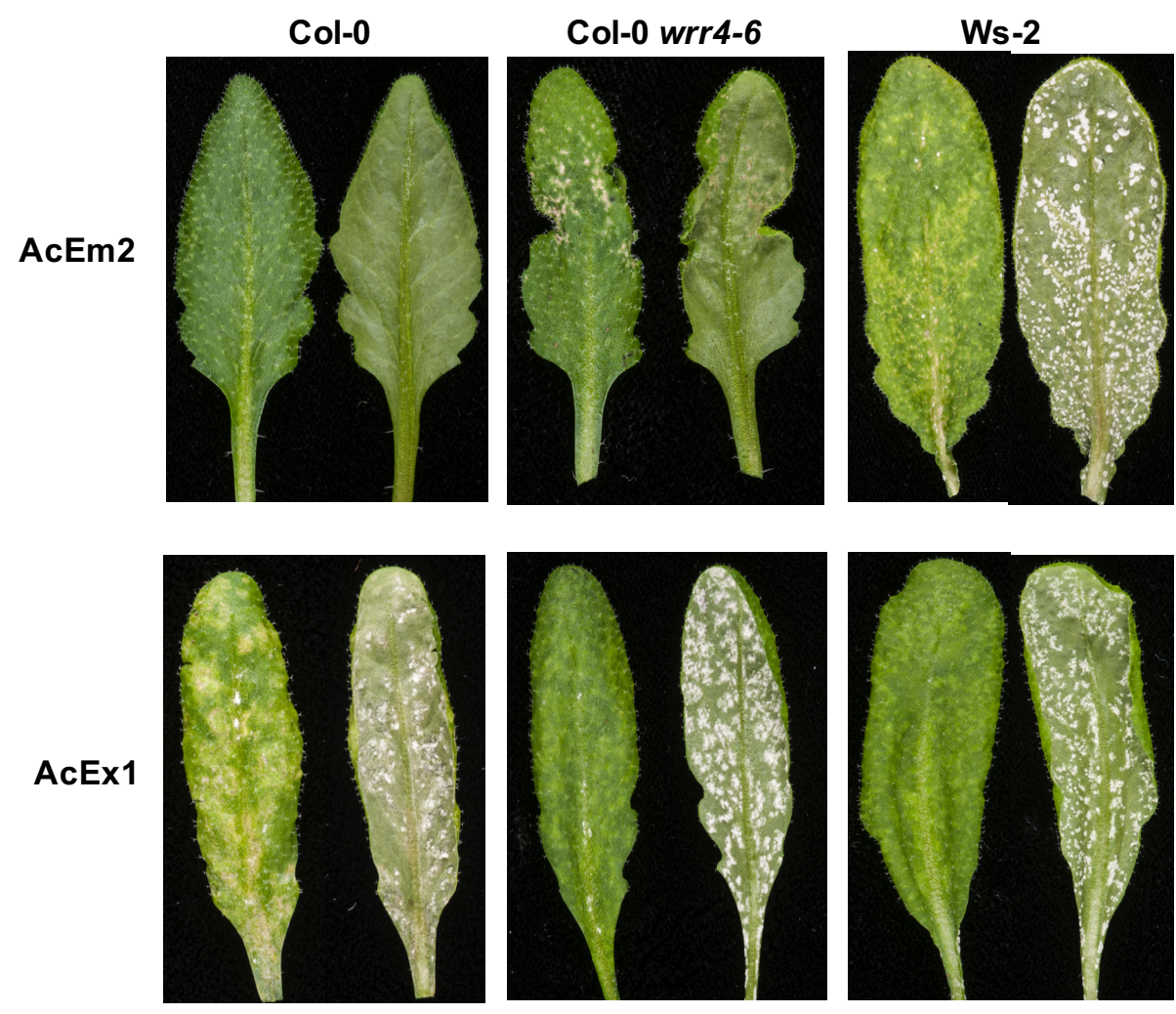

Figure 1. Resistant and susceptible phenotypes of $W R R 4 A^{\text {Col-0 }}$ A. thaliana following inoculation of adult leaves with two different isolates of $A$. candida Race 4.

Arabidopsis Col-0 is resistant to Race 4 isolate AcEm2 (collected from Capsella bursa-pastoris) and susceptible to Race 4 isolate AcEx1 (collected from A. halleri) whereas Ws-2 is susceptible to both

310 isolates. Necrotic resistance observed with the Col-0-wrr4-6 mutant indicates that WRR4A is responsible for full green resistance to AcEm2. Five-week old plants were spray-inoculated with $A$. candida and incubated at $21^{\circ} \mathrm{C}$; and then phenotyped 14 days after inoculation. Abaxial and adaxial picture of the same leaf are shown. Scale $=1 \mathrm{~cm}$.

315 Selection of candidate $\boldsymbol{A}$. candida CCG effectors to test for recognition by different WRR4 paralogs

The recent PacBio based genome assembly of $A$. candida Race 2 isolate Ac2V ("Ac2VPB") revealed that the CCG family of secreted proteins corresponds to ca. $10 \%$ of the A. candida secretome (Furzer et al., 2021). The genome of each A. candida race contains 60-80 CCG effector candidate genes that can vary by sequence and presence/absence polymorphism (Jouet et al., 2019; Furzer et al., 2021). This enabled identification of candidates for functional screening of CCG effectors transiently, testing for WRR gene-dependent HR. Such transient assays have been adopted for other oomycete pathogens like $P$. infestans, testing secreted proteins with an RxLR motif

325 (Vleeshouwers et al., 2008; Vleeshouwers et al., 2011), and thus, identifying several recognized effector genes. 
To identify CCG effector proteins that are recognized by WRR4 paralogs, we analysed CCGs predicted from Illumina-based genome assemblies of multiple $A$. candida races (AcNc2, AcEm2, Ac7V, AcBoT and Ac2V) (McMullan et al., 2015; Jouet et al.,

330 2019) as well as additional CCGs predicted from Ac2VPB (Furzer et al., 2021). We identified CCGs showing presence/absence polymorphism or pseudogenisation (Jouet et al., 2019; Furzer et al., 2021). We then selected 30 candidate CCGs to screen with $W R R 4 A$ prioritising those either absent or pseudogenised in WRR4A-overcoming race AcEx1. To screen with WRR4B, we selected 13 candidate CCGs that are mostly 335 conserved in $\mathrm{Ac} 2 \mathrm{~V}, \mathrm{Ac7V}$ and AcBoT but are absent or pseudogenised in other $A$. candida races (Fig. 2a,b and Fig. 3a; Table S3).

To test the selected CCG effectors for $W R R 4 A$ or $W R R 4 B$ recognition, we cloned CCG effectors into an expression vector with $35 \mathrm{~S}$ promoter and transformed into Agrobacterium for infiltration of $N$. tabacum or N. benthamiana leaves. The transient co-

340 delivery of CCG effector was performed either with GFP or RFP as negative control and with WRR4A or WRR4B (Cevik et al., 2019). Effectors that trigger an HR when coexpressed with the corresponding NLR were further validated by the luciferase eclipse assay modified from Allen et al., (2004) for recognition in Col-0 or in Col-0 wrr4a or $w r r 4 b$ mutants (Fig. 2a).

\section{$W R R 4 A^{\text {Col-0 } 0}$ confers recognition to eight different $C C G$ effectors from $A$. candida}

To screen candidate CCGs for their recognition by WRR4A, CCGs excluding the signal peptide were cloned from isolates of $A$. candida Race 2 (Ac2V) or Race 4 (AcEm2 or AcNc2). All of the effector alleles were co-infiltrated with $35 \mathrm{~S}: W R R 4 A$-expressing 350 Agrobacterium strains (Cooper et al., 2008; Cevik et al., 2019). All of the recognized effectors and some representative non-recognized CCGs were tested by Western blot to confirm their protein expression when expressed transiently (Fig. S1). Among the 30 alleles we tested, eight CCGs (CCG28 $8^{\mathrm{Ac} 2 \mathrm{~V}}, \mathrm{CCG} 30^{\mathrm{AcNc} 2}, \mathrm{CCG}^{\mathrm{AcNc} 2}, \mathrm{CCG} 40^{\mathrm{AcEm} 2}$, $\mathrm{CCG} 67^{\mathrm{AcEm} 2}, \mathrm{CCG} 71^{\mathrm{AcNc} 2}, \mathrm{CCG} 79^{\mathrm{Ac} 2 \mathrm{~V}}$ and $\mathrm{CCG} 104^{\mathrm{Ac} 2 \mathrm{~V}}$ ) elicited HR within 36-48 hours

355 post infiltration (hpi) when co-expressed with WRR4A but not with GFP control. Moreover, WRR $4 A$ co-expressed with GFP did not show any autoactivation (Fig. 2c).

To validate the HR observed from transient assays with the eight recognized CCGs, we carried out luciferase eclipse assays by transient delivery using particle bombardment to reveal reduced luciferase activity upon HR triggered by these recognized 
(a)

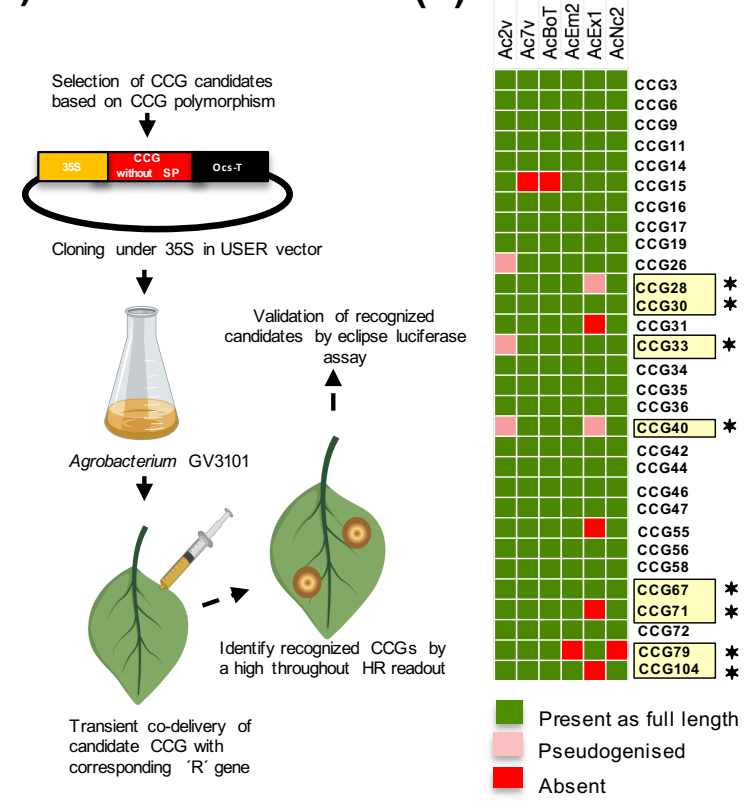

(c)

(d)
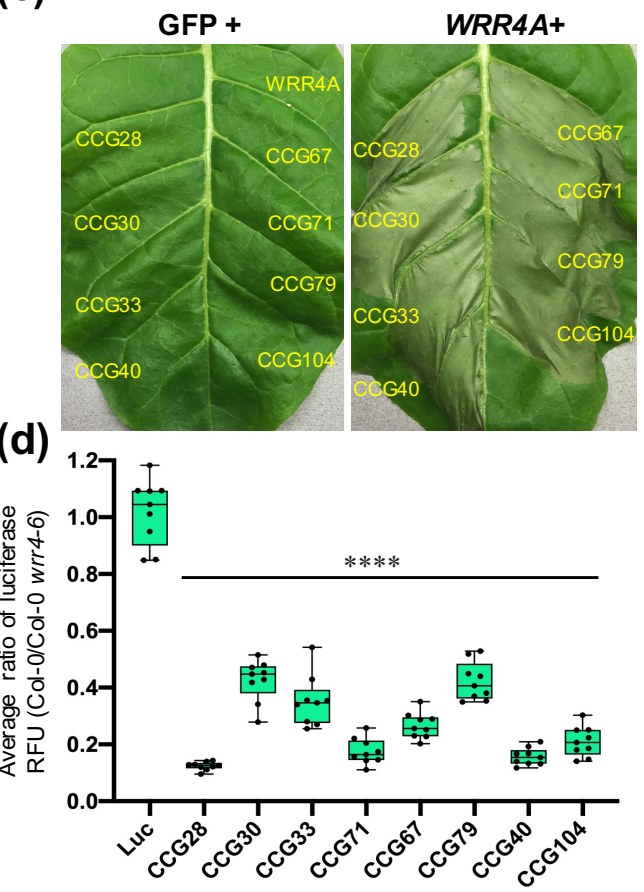

Figure 2. Identification of eight CCG effectors from $A$. candida that elicit hypersensitive response when co-expressed in tobacco leaves with $W R R 4 A$ from Col-0 $A$. thaliana.

(a) Pipeline for high-throughput screening of the CCG effectors for identification of recognized-CCGs against $W R R 4 A^{\mathrm{Col}-0}$ and $W R R 4 B{ }^{\mathrm{Col}-0}$. The selected candidate effectors without the signal peptide (SP) were cloned into a USER expression vector under CaMV-35S promoter for Agrobacterium-mediated transient expression either in $N$. tabacum or $N$. benthamiana. Recognized CCGs were identified by an HR readout and were further validated by a luciferase eclipse assay.

(b) The selection of candidate CCG effectors for screening against WRR $4 A$ was based on positive selection, presence across different $A$. candida races and/or showing the characteristic of either allelic truncation or absence in a Col-virulent isolate of Race 4 (AcEx1). The recognized CCGs against WRR4A identified from our screen are highlighted with a yellow box and marked with an asterisk $(*)$.

(c) Transient expression of candidate CCG effectors either with GFP or with WRR $4 A^{\mathrm{Col}-0}$ in N. tabacum.

The eight identified CCGs trigger HR when co-expressed with $W R R 4 A^{\mathrm{Col}-0}$ but not with GFP alone. $W R R 4 A^{\text {Col-0 }}$ also do not show any auto-activity when co-delivered with the GFP control.

(d) The WRR4A-dependent CCG recognition correlates with the luciferase eclipse assay upon particle bombardment in $A$. thaliana. Graph showing a drop in the luciferase activity of all recognized CCGs identified by a ratio of the measured luciferase in wildtype Col- 0 as compared to that Col-wrr $4 a-6$ mutant validating the recognition to be specific by $W R R 4 A^{\text {Col- } 0}$. Statistical significance versus Luciferase (Luc) alone ( $p<0.05$, one way ANOVA, Bonferroni's multiple comparison test) is indicated by an asterisk. Error bars indicate $\mathrm{SD}(\mathrm{n}=9)$.

luciferase construct was delivered alone or with recognized CCG effector and incubated in vials with water for 48 hours post-bombardment (hpb). All the CCGs recognized by WRR $4 A$ conferred reduced luciferase activity in comparison to the luciferase only control in Col-0 but not in Col-0 wrr4a-6 mutant. The diminished luciferase activity in Col-0 380 indicates HR-dependent cell death in transformed leaf cells (Fig. 2d). Hence, both methods revealed eight different CCGs that are recognized by WRR $4 A$. 


\section{The $W R R 4 A^{C o l-0}$ paralog $W R R 4 B^{C o l-0}$ recognizes four additional CCG effectors}

To test recognition by WRR4B, we selected candidate CCGs from the CCG effectorome repertoire based on the resistance and susceptibility disease phenotypes (Table S2). As

$385 W R R 4 B$ confers resistance against $A$. candida race $2(\mathrm{Ac} 2 \mathrm{~V})$, we prioritized $13 \mathrm{CCG}$ candidates present mainly in crop-infecting races of $A$. candida and also CCGs present exclusively in Ac2V (Fig. 3a), selecting the Ac2V CCG allele for these recognition assays.

Over-expression of $W R R 4 B$ in tobacco or $N$. benthamiana shows weak 390 autoimmunity, even when infiltrated with the GFP control (Fig. S2 a,b). This creates a requirement for cautious interpretation of any HR phenotypes detected upon coexpression with a potentially recognized CCG effector. Hence, we used the luciferase eclipse assay for screening the set of 13 cloned CCGs for candidate WRR $4 B$-recognized CCGs, and discovered four candidate $W R R 4 B$ - recognized CCGs. We further confirmed

395 the recognition of these four candidates $\left(\mathrm{CCG} 45^{\mathrm{Ac} 2 \mathrm{~V}}, \mathrm{CCG} 57^{\mathrm{Ac} 2 \mathrm{~V}}, \mathrm{CCG} 61^{\mathrm{Ac} 2 \mathrm{~V}}\right.$ and CCG70 ${ }^{\mathrm{Ac} 2 \mathrm{~V}}$ ) by luciferase eclipse assay by a comparison of the luciferase activity in Col0 and Col-0-wrr4b mutant. The Col-0 wrr4b mutant showed a higher level of luciferase when co-expressed with these 4 CCGs that is diminished in Col-0 due to HR (Fig. 3b). This assay further confirms the $W R R 4 B$-specific recognition of these four CCGs from $A$. 400 candida race Ac2V .To understand if any of these CCGs confer elevated HR after transient expression in N. tabacum compared to WRR4B alone, CCG45 ${ }^{\mathrm{Ac} 2 \mathrm{~V}}, \mathrm{CCG} 57^{\mathrm{Ac} 2 \mathrm{~V}}$, CCG61 $1^{\mathrm{Ac} 2 \mathrm{~V}}$ and CCG70 ${ }^{\mathrm{Ac} 2 \mathrm{~V}}$ were co-infiltrated with $W R R 4 B$. Only CCG45 $5^{\mathrm{Ac} 2 \mathrm{~V}}$ and CCG70 ${ }^{\mathrm{Ac} 2 \mathrm{~V}}$ showed an enhanced HR compared to the WRR 4B infiltrated with GFP alone, indicating that these candidates are also recognized by $W R R 4 B$ in tobacco (Fig. S2a). This 405 HR varied between individual leaves. CCG57 $7^{\mathrm{Ac} 2 \mathrm{~V}}$ and CCG61 ${ }^{\mathrm{Ac} 2 \mathrm{~V}}$ did not show elevated HR compared to WRR $4 B$ co-infiltrated with GFP control (Fig. S2a). In order to check if the HR by $\mathrm{CCG} 45^{\mathrm{Ac} 2 \mathrm{~V}}$ and $\mathrm{CCG} 70^{\mathrm{Ac} 2 \mathrm{~V}}$ is also consistent in $N$. benthamiana, we performed co-infiltration of these CCGs with $W R R 4 B$ in N. benthamiana. CCG $28^{\mathrm{Ac} 2 \mathrm{~V}}$ codelivered with WRR4A was used as a positive control. Consistent with N. tabacum

410 phenotype, CCG45 ${ }^{\mathrm{Ac} 2 \mathrm{~V}}$ and CCG70 ${ }^{\mathrm{Ac} 2 \mathrm{~V}}$ show an elevated HR in N. benthamiana when co-expressed with $W R R 4 B$ as compared to $W R R 4 B$ alone (Fig. S2b, c). The CCG motif region is the most conserved part of different WRR $4 B$-recognized CCGs (Fig. 3c). We conclude that $W R R 4 B$ specifically recognizes four CCGs from $A$. candida that are distinct from the CCGs that are recognized by $W R R 4 A$. 

WRR4A-CCG interaction, as WRR $4 A$ does not show any autoimmune phenotype and also recognizes diverse CCGs across different $A$. candida clades (Furzer et al., 2021).

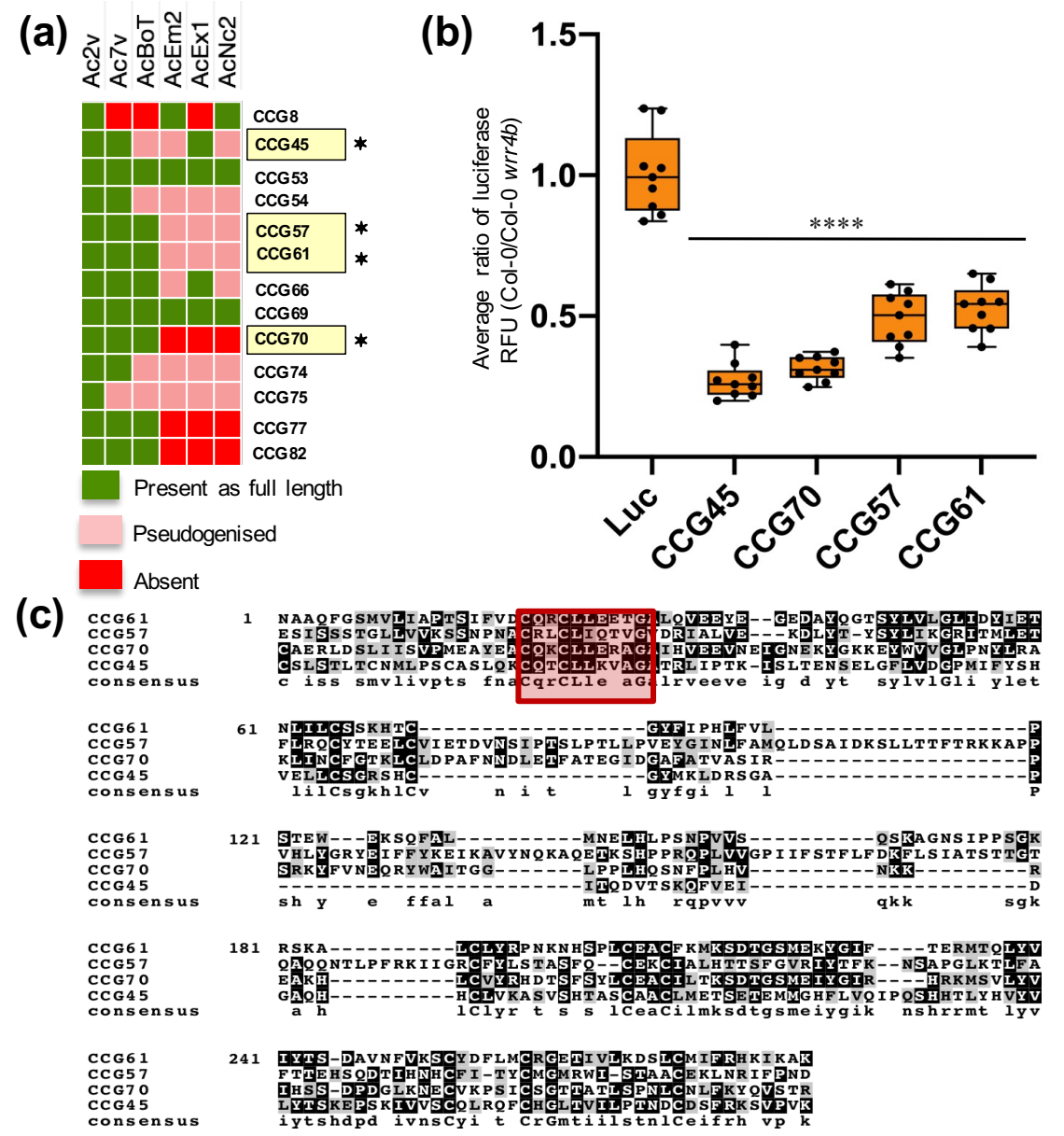

420 Figure 3. The $W R R 4 B^{\text {Col-0 }}$ paralog recognizes four additional CCG effectors in $A$. candida.

(a) The selection of candidate CCG effectors for screening against $W R R 4 B{ }^{\mathrm{Col}-0}$ based on their presence across isolates of different $A$. candida races. The recognized CCGs against $W R R 4 B{ }^{\mathrm{Col}-0}$ identified from our screen are marked with an asterisk $(*)$.

(b) The luciferase eclipse assay upon particle bombardment in A. thaliana. Graph showing a drop in the luciferase activity of all recognized CCGs identified by a ratio of the measured luciferase in wildtype Col-0 as compared to a Col-wrr $4 b$ mutant validating the recognition to be specific by $W R R 4 B^{\mathrm{Col}-0}$. Statistical

425 significance versus Luciferase (Luc) alone ( $p<0.05$, one way ANOVA, Bonferroni's multiple comparison test) is indicated by an asterisk. Error bars indicate $\mathrm{SD}(\mathrm{n}=9)$.

(c) Multiple sequence alignment of $W R R 4 B$-recognized CCGs which shows homology primarily in the CCG motif. CCG motif is highlighted by red box.

\section{Recognition of CCGs by $W R R 4 A^{\mathrm{Col}-0}$ requires the $\mathrm{N}$-terminal portion of the protein}

430 but does not occur via the CCG motif

To investigate the mechanism of $W R R 4 A$ activation by CCGs, we tested a series of CCG effector deletions. We selected CCG28 for this analysis because it triggers the strongest 
WRR $4 A$-dependent HR, with a response visible at 36 hpi. Similar to WRR $4 B$ - recognized CCGs, as the CCG motif region was the only part of the CCG protein that showed 435 homology across different $W R R 4 A$ recognized CCGs, we tested the role of the N-terminal region of CCG for recognition by $W R R 4 A$. A full-length version of CCG28 without its secretion signal triggers strong $W R R 4 A$-dependent HR. However, deletion of amino acids 28-40 in the N-terminal region after the secretion signal abolishes this recognition (Fig. 4a, b).

440 To define the minimal N-terminal region of CCG28 that is recognized, we made Cterminal deletions of $\mathrm{CCG} 28^{\mathrm{Ac} 2 \mathrm{~V}}$ and tested their recognition using transient assays in $N$. tabacum leaves (Fig. S3). A truncation of CCG2 $8^{\mathrm{Ac} 2 \mathrm{~V}}$ that includes the first 100 amino acids after the signal peptide site $\left(\mathrm{CCG} 28^{28-130}\right)$, including the CCG motif, is more strongly recognized by $W R R 4 A$ than full length CCG28, triggering an elevated HR at 36

445 hpi when transiently co-expressed in N. tabacum (Fig. 4a, b; Fig. S3). This also includes a common feature with a pair of cysteines located $\sim 50$ amino acids after the CCG motif. In contrast, a C-terminal region of CCG28 without the CCG motif, which corresponds to aa 56-543 abolishes recognition when co-expressed with $W R R 4 A$ (Fig. 4a, b; Fig. S4). Hence, we conclude, that the truncated N-terminal part of CCG28 is indispensable for WRR $4 A$ recognition. Further deletion of amino acids 28-35 at the N-terminus also compromises recognition (Fig. S3 and S5). To define the shortest region of CCG28 that is recognized, we further narrowed the recognition region to 50 amino acids, corresponding to CCG28 $8^{28-78}$. However, only YFP-tagged versions of this shortest region activate HR (Fig. S3 and S5), likely indicating that untagged versions are insufficiently stable for their interaction with $W R R 4 A$ to trigger a cell death phenotype when expressed transiently.

We next tested whether WRR4A recognition of all the CCG candidates involves their N-termini. A truncation analysis was carried out for all the eight recognized CCGs by WRR4A. Upon transient expression, C-terminally truncated versions of the other seven 460 recognized CCGs also trigger an HR phenotype with WRR4A in N. tabacum (Fig. S6a). These data suggest that the N-terminal portion of all the $A v r-W R R 4 A$ is sufficient for recognizability. WRR $4 A$ recognition of CCGs requires an intact Walker A (P-loop) (Schreiber et al., 2016) because a mutation in this P-loop of WRR4A with a change from K220 >L220 abolishes the HR when WRR4A is co-infiltrated with the recognized CCGs 465 in transient assays (Fig. S6b). 
(a)

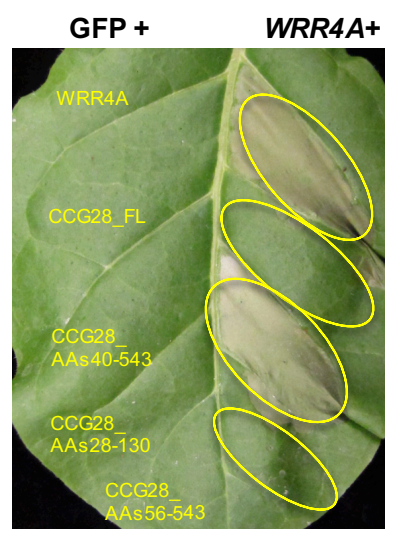

(c) (b)

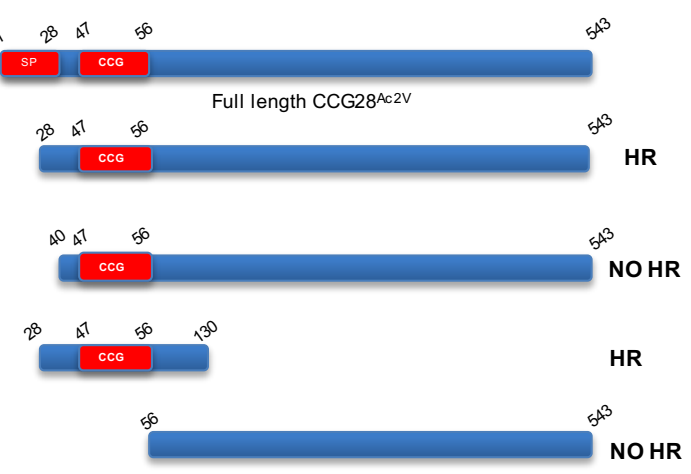

(d)
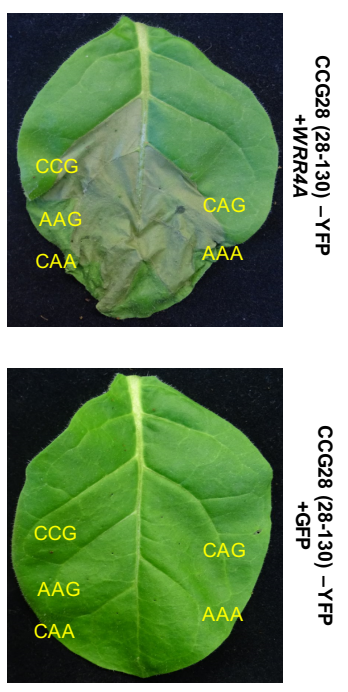

Figure 4. The N-Terminal Portion of CCG effectors is sufficient for recognition by $W R R 4 A^{\mathrm{Col}-0}$ and occurs independent of the CCG motif

(a) Transient expression of different truncated versions of CCG28 either with GFP or with $W R R 4 A^{\mathrm{Col}-0}$ in

N. tabacum. The N-terminal region that corresponds to aa 28-130 is mainly indispensable for WRR4A ${ }^{\mathrm{Col}-0}$ recognition.

(b) Schematic representation of all the truncations infiltrated in Fig. 4a, showing either a HR or a No-HR phenotype.

(c) The alignment of protein sequences of $\mathrm{N}$-terminal region of CCGs after signal peptide cleavage, up to amino acid 130 in all WRR4A ${ }^{\text {Col-0 }}$ recognized CCGs. CCG motif is highlighted by red box.

475 (d) Mutations in CCG motif with CCG changed to AAG, CAA, CAG and AAA do not impair WRR4A ${ }^{\mathrm{Col}-0}$ recognition and HR. N. tabacum leaf was infiltrated to express wild-type or mutated variants of CCG motif in CCG28 ${ }^{\text {a228-130 }}$-YFP. All versions with wild-type as well as mutated CCG motif trigger HR, which was assessed at 48 hpi. Photographs are representative of three consistent replicates.

We next examined whether the previously defined CCG motif, that shows 480 maximum homology across all recognized CCGs against $W R R 4 A$ mediates effector

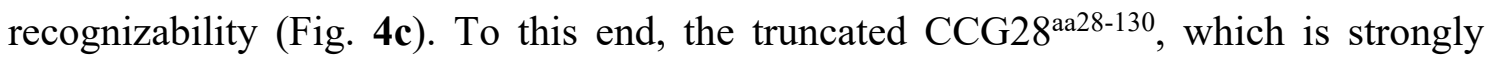

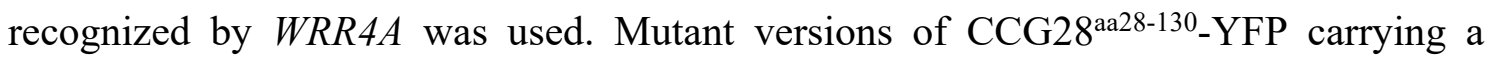
mutation in the CCG motif (where CCG exchanged to AAG, CAA, CAG and AAA) were generated and tested for recognition in transient assays by co-infiltration with $W R R 4 A$ in

$485 N$. tabacum. These mutated versions were still strongly recognized by $W R R 4 A$ with an HR phenotype indistinguishable from the truncated version with authentic CCG motif (Fig. 4d). These data suggest that $W R R 4 A-\mathrm{CCG}$ recognition does not occur via the CCG motif. Conceivably a structural similarity in the N-terminal portion of these recognized 
CCGs might be responsible for their detection by WRR4A. Consistent with this, CCG30, but not its close paralog CCG16, is recognized by WRR4A (Fig. S7).

\section{Allelic variation and expression polymorphism of recognized WRR4A CCGs}

Next, we confirmed the allelic status of the identified recognized CCG candidates across all the sequenced races of $A$. candida from the available Illumina assemblies of different 495 A. candida races (McMullan et al., 2015) and the recent PacBio assembly of B. junceainfecting race Ac2V (Furzer et al., 2021). Consistent with the resistance phenotypes in Col-0 and Col-wrr4a mutants, the recognized CCGs are present across different $A$. candida races. CCG33 and CCG40 are pseudogenised in the B. juncea-infecting race Ac2V (Fig. 2b). Several of the recognized alleles from these CCG candidates, show

500 polymorphism in AcEx1 (a WRR4 resistance-breaking pathotype of Race 4). Due to an early stop codon, CCG28 $8^{\text {AcEx1 }}$ shows a protein length of only 226 amino acids as compared to the full-length alleles from other races (Fig. 5a). Another isolate of this pathotype, AcCarlisle collected on A. thaliana, shows the same early stop codon in CCG28 and virulence on Arabidopsis Col-0. The AcEx1 CCG28 allele is weakly 505 recognized compared to full-length alleles from $A$. candida Races 2, 4 and 7 (Ac2V, AcEm2 and Ac7V) all of which trigger an early WRR4A-dependent HR at 36 hpi (Fig. 5b). Additionally, the WRR4A-recognized CCG71 and CCG104 are absent from AcEx1 (Fig. 2b) suggesting that absence of these effectors might contribute to evasion of $W R R 4 A$ mediated resistance. On the other hand, the CCG effectors recognized by WRR4B showed

510 a strong presence/absence polymorphism and are primarily present in the crop-infecting races which are known to be resisted by $W R R 4 B$ (Fig. 3a).

To determine the extent to which these WRR $4 A$ - and WRR $4 B$-recognized CCGs are expressed in planta, we assessed their expression profiles from the RNA-Seq data obtained over the consecutive time-points during serial infection stages of $A$. candida

515 Race Ac2V (Furzer et al., 2021). WRR4A- and WRR4B-recognized CCGs in Ac2V show in planta induction during different colonization stages. These CCGs are induced specifically at different colonization time-points of 2, 4 and 6 dpi (Fig. S8a) consistent with a role in virulence. 
(a)

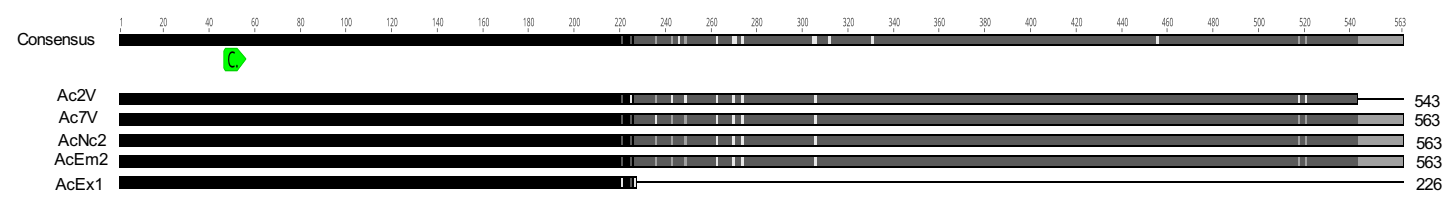

(b)

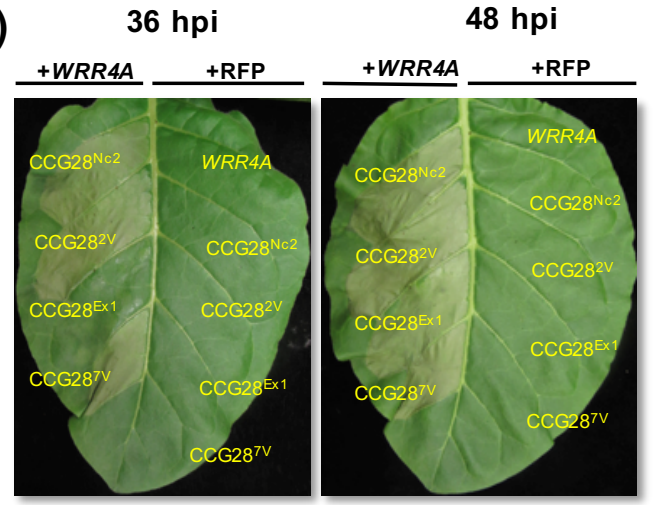

(c)

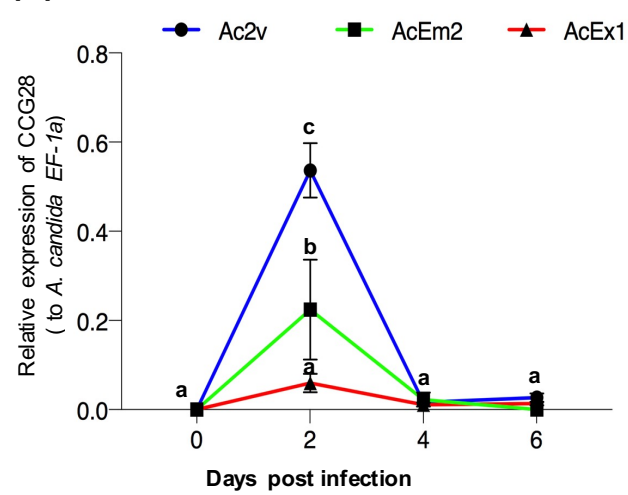

$520 \quad$ Figure 5. Differential recognition of CCG28 alleles across different $\boldsymbol{A}$. candida races.

(a) Schematic representation of the amino acid sequence alignment of CCG28 alleles from A. candida Race 2 (Ac2V), Race7 (Ac7V) and Race 4 (AcNc2, AcEm2 and AcEx1). The black region indicates conserved amino acid sequence across all races, whereas the grey region indicates variation. White bars in the alleles indicate the polymorphic sites where there is no predominant residue, and grey bars indicate polymorphic sites where there is a predominant residue. The numbers next to the alleles refer to the total protein length. Figure drawn to scale. The CCG motif is indicated in green with a $\mathrm{C}$.

(b) Quantitative recognition of different CCG28 alleles in $N$. tabacum transient assay. The different CCG28 alleles were co-infiltrated with WRR4A ${ }^{\text {Col-0 }}$ into $N$. tabacum leaves. CCG28 shows recognition with an enhanced HR at 36 hpi. However, the AcEx1 CCG28 allele with a premature stop codon shows a delayed HR at $48 \mathrm{hpi}$, consistent with weak recognition of the CCG28 allele in an A. candida Race 4 isolate that overcomes the WRR $4 A^{\mathrm{Col}-0}$ recognition.

(c) Expression analysis of CCG28 (relative to A. candida EF-1a) in Race 2 (Ac2v) and Race 4 (AcEm2 and AcEx1), showed that the AcEx1 truncated allele of CCG28 is weakly expressed, likely contributing to virulence on $W R R 4 A^{\mathrm{Col}-0}$. Different letters indicate statistically significant differences between the different alleles tested ( 2 way ANOVA, Bonferroni's multiple comparison test, $\mathrm{p}<0.05$ ). Error bars represent SD.

To investigate the expression patterns of different alleles of the WRR $4 A$ recognized CCGs, we performed a qRT-PCR expression profiling of the $A$. candida races Ac2V, AcEm2 and AcEx1 growing on Ws-eds1 plants (Fig. S8b). These data showed

535 different $\mathrm{CCG}$ effectors increase in expression during infection. Intriguingly, CCG28 is primarily induced at $2 \mathrm{dpi}$. The Ac2V allele of CCG28 is most highly expressed followed by the AcEm2 allele. Notably, the pseudogenised allele from AcEx1 shows least expression as compared to the other alleles (Fig. 5c). The expression profiles of other recognized $W R R 4 A$ - recognized CCGs follow a similar trend. The Ac2 V allele is among the most highly expressed and AcEx1 shows the lowest (Fig. S8c). From these results, 
we conclude that the partial susceptibility of Col-0 plants we observe with AcEx1 is both due to reduced expression or due to absence of the WRR4A-recognized CCGs. In summary, these data suggest for WRR4-recognized CCGs, there exists expression polymorphism as well as presence-absence polymorphism that can enable evasion of

545 resistance, especially when combined with the well-documented suppression of resistance upon Albugo infection.

\section{AcEx1 has the capacity to suppress $W R R 4 A$-mediated resistance}

Previous studies have shown that $A$. candida infection leads to strong immune suppression enabling co-infection by otherwise avirulent races, permitting sexual exchange and recombination (Cooper et al., 2008; McMullan et al., 2015). To test if AcEx1 can suppress $W R R 4 A$ mediated resistance, we performed sequential inoculation experiments, on Arabidopsis accessions with and without $W R R 4 A$, monitoring A. candida races using race-specific genomic regions as a readout for race-specific PCR markers.

555 Race-specific PCR verifies that AcNc2 can grow on Ws-2 which does not have WRR4A but not on RIL CW20 (which carries Col-5 alleles of WRR $4 A$ and WRR $4 B$ as the only WRR genes). However, pre-inoculated plants of CW20 colonized by the WRR $4 A$ resistance breaking race AcEx1 lose resistance in CW20 leaves towards AcNc2 (Fig. S9). Therefore, AcEx1 not only suppresses WRR $4 A$ mediated recognition but also enables

560 other races to grow, that would otherwise be resisted. These data suggest that AcEx1 is able to overcome $W R R 4 A$ resistance by virtue of weak recognizability due to polymorphisms in its repertoire of $W R R 4 A$ recognized CCG alleles, together with strong ability for immunosuppression of $W R R 4 A$-mediated resistance.

\section{DISCUSSION}

Oomycetes in the Peronosporales (Phytophthora, Pythium and downy mildew species) are destructive pathogens. Host colonization requires effectors which carry a signal peptide and a positionally constrained RxLR motif (Win et al., 2006). A specific effector is often detected in a resistant host by a matching NLR immune receptor, often encoded by a resistance $(R-)$ gene, that activates a defense response that thwarts pathogen success (Armstrong et al., 2005; Rehmany et al., 2005). The Irish potato famine pathogen $P$. infestans, RxLR effectors Avrblb2 and Avrvnt1 are recognized by Rpi-blb2 and Rpi-vnt1 (Oh et al., 2009; Pais et al., 2018). Identifying such avirulence (Avr) determinants in the pathogen is important for improving strategies to develop disease resistant crops. 
575 The genomes of oomycetes in the Albuginales, such as A. laibachii and A. candida, are not enriched for RxLR proteins but instead contain a family of secreted proteins with a ' $\mathrm{CHxC}$ ' (now CCG) motif that contributes to translocation (Kemen et al., 2011; Furzer et al., 2021). These proteins show signatures of diversifying selection, with high ratios of non-synonymous to synonymous mutations, similar to RxLR effectors 580 (Rehmany et al., 2005; Asai et al., 2018). The Ac2VPB assembly of the Brassica pathogen $A$. candida Race 2 has enabled refinement of CCG effector annotation (Furzer et al., 2021). CCG gene repertoires are present in all sequenced $A$. candida races (McMullan et al., 2015). A combined phylogeny comparison between A. laibachii and A. candida shows an expansion of CCGs from $\sim 30$ in A. laibachii to around $\sim 100$ in $A$.

585 candida, with several $A$. candida-specific clades suggesting expansion and reshuffling of CCG effector repertoires to adapt to different hosts (Furzer et al., 2021). Moreover, the CCG repertoire displays elevated rates of pseudogenisation and presence/absence polymorphisms, consistent with selection for diversity while maintaining virulence functions.

In this study, we used the CCG effectoromes from different $A$. candida races, enabling us to screen selected CCG effectors for $W R R 4$ recognition. This revealed eight WRR4A-recognized CCGs and four WRR4B-recognized CCGs. The allelic comparison approach helped us to select CCG candidates based on their presence/absence polymorphism in A. candida races that overcome resistance. The eight identified $W R R 4 A$ 595 recognized CCGs when co-expressed with $W R R 4 A$ show an HR at 48 hpi resulting in complete cell-death. A genetic validation of this recognition by luciferase eclipse assay after particle bombardment shows that the identified CCG effectors are specifically recognized by $W R R 4 A$. A similar approach based on allelic comparison allowed us to screen and identify four additional WRR4B-recognized CCGs. As WRR $4 B$ shows an 600 autoimmune phenotype in transient expression experiments, the $N$. tabacum HR assay was more difficult to interpret than with $W R R 4 A$. The luciferase eclipse assay enabled us to identify four WRR4B-recognized CCGs; CCG45, CCG70, CCG57 and CCG61. However, only CCG45 and CCG70, which show the strongest phenotype in the luciferase eclipse assay show an enhanced HR when co-expressed transiently with $W R R 4 B$ in $N$.

605 tabacum or $N$. benthamiana. We conclude that $W R R 4 A$ and $W R R 4 B$ each recognize multiple and distinct CCG effectors from A. candida. Multiple recognition of effectors is known for some other NLRs that function in pairs, such as Arabidopsis RPS4/RRS1 (Sarris et al., 2015; Guo et al., 2020) and rice RGA4/RGA5 (Cesari et al., 2013). The 
WRR $4 B$ autoimmune phenotype hindered further analysis, and we further investigated the

610 WRR4A-CCG interaction to define recognition requirements in more detail.

Analysis of the recognition mechanism of multiple CCGs by WRR $4 A$ revealed that the N-terminal portion of all WRR $4 A$-recognized CCGs is sufficient for recognizability. Truncation analysis of all these CCGs shows the $\mathrm{N}$ terminal 12 amino acids after the secretion signal are required to trigger HR. Mutations in the CCG motif

615 suggest that the CCG motif itself is not recognized, as mutant alleles are still recognizable. Hence, the exact role of the CCG motif and its significance in A. candida infection is still unclear. The recognized CCGs fall into different clades in the CCG phylogeny (Furzer et al., 2021). Hence, we speculate that there likely exists a structural similarity among these recognized effectors that enables their recognition. This is also suggested by the 620 observation that CCG16, a close paralog of the WRR4A-recognized CCG30 is not recognized despite showing a higher homology to CCG30 than some other WRR4Arecognized CCGs.

This study provides evidence that WRR $4 A$ and $W R R 4 B$ at the WRR4 locus in $A$. thaliana Col-0 have the capacity to recognize multiple effectors from $A$. candida likely 625 making them highly effective for immune activation upon pathogen attack. This potentially explains the effectiveness of this resistance against many races. Interestingly, both $W R R 4 A$ and $W R R 4 B$ have a post-LRR C-JID domain which was recently shown to physically interact with effectors by TNLs RPP1 and Roq1 during resistosome activation (Ma et al., 2020; Martin et al., 2020). Hence, it will be intriguing to understand whether 630 WRR4-CCG interaction is mediated by a similar mechanism. For P. infestans RxLR effectors, the C-terminal post-RxLR domain carries their effector activities (Kamoun, 2006; Kamoun, 2007). It is unknown how CCG proteins contribute to pathogen virulence and is a topic of further investigation. AcEx1 is capable of overcoming WRR $4 A$ - and WRR $4 B$-mediated resistance, though on Col-0, but not Col-0 wrr $4 A$, it activates chlorosis

635 and thus is still weakly recognized. Its growth on Col-0 is due to the loss of some of the recognized $A$. candida effectors, and reduced expression of others.

Additionally, AcEx1 suppresses WRR4-mediated immunity against AcNc2, consistent with the hypothesis that Arabidopsis susceptibility to a specific $A$. candida race is determined by a balance of effector detection and effector-mediated suppression of 640 defense. The partial susceptibility of Col-0 plants with AcEx1 is potentially explained by the reduced expression (measured by expression profiling) of otherwise detectable CCG effectors in this isolate. We suggest that strong immunosuppression of this weak 
recognition enables AcEx1 to colonize Col-0. Therefore, we propose that further study of $W R R 4 A$ - and WRR $4 B$ - recognized CCGs might reveal insights into the colonization 645 biology of $A$. candida.

Resistance to AcEx1 that maps to the WRR4 locus has been identified in other accessions of Arabidopsis (Fairhead, 2016; Castel et al., 2021). The WRR4A alleles in HR-5 and Oy-0 have an extended C-terminus, that confers AcEx1 resistance by recognizing alternative CCG effectors. The identification of WRR $4 A$ - and $W R R 4 B$ recognized CCGs will further enable understanding of the recognition mechanism at the structural level. Thus, this work contributes new insights into effector biology in obligate biotrophs, and will help inform provision of durable resistance in Brassicaceae crops by pyramiding or transgene stacking of different WRR4 paralogs which recognize diverse repertoires of CCG effectors.

\section{Author Contributions}

A.R., V.C. and J.D.G.J conceptualized and designed the research. A.R., V.C. and K.B. S.F. conducted all experiments. A.R., V.C., K.B., O.J.F. and S.F. performed the data analysis. M.H.B. and E.H gave critical intellectual input and provided material for this work. A.R., V.C. and J.D.G.J wrote the manuscript with input from all co-authors. All authors helped editing and finalizing the manuscript.

\section{Acknowledgements}

A.R. acknowledges support by EMBO LTF (ALTF-842-2015). V.C., O.J.F. and S.F. were supported by Biotechnology and Biological Sciences Research Council (BBSRC) grant BB/L011646/1. K. B. and J.D.G.J. were supported in part by ERC Advanced Investigator grant to JDGJ 'ALBUGON' Project ID 233376. Research in the Jones Lab is supported by the Gatsby Foundation (UK) and BBSRC. We thank Shihomi Uzuhashi for her help in initial screening of the Albugo effectors in tobacco.

\section{References}

Allen RL, Bittner-Eddy PD, Grenville-Briggs LJ, Meitz JC, Rehmany AP, Rose LE, Beynon JL. 2004. Host-Parasite Coevolutionary Conflict Between Arabidopsis and Downy Mildew. Science 306(5703): 1957-1960.

Armstrong MR, Whisson SC, Pritchard L, Bos JIB, Venter E, Avrova AO, Rehmany AP, Böhme U, Brooks K, Cherevach I, et al. 2005. An ancestral oomycete locus contains late blight avirulence gene Avr3a, encoding a protein that is recognized 
in the host cytoplasm. Proceedings of the National Academy of Sciences of the United States of America 102(21): 7766.

Asai S, Furzer OJ, Cevik V, Kim DS, Ishaque N, Goritschnig S, Staskawicz BJ, Shirasu K, Jones JDG. 2018. A downy mildew effector evades recognition by polymorphism of expression and subcellular localization. Nature Communications 9(1): 5192.

685 Belhaj K, Cano LM, Prince DC, Kemen A, Yoshida K, Dagdas YF, Etherington GJ, Schoonbeek H-j, van Esse HP, Jones JDG, et al. 2017. Arabidopsis late blight: infection of a nonhost plant by Albugo laibachii enables full colonization by Phytophthora infestans. Cellular Microbiology 19(1): e12628.

Borhan MH, Gunn N, Cooper A, Gulden S, Tör M, Rimmer SR, Holub EB. 2008. WRR4 Encodes a TIR-NB-LRR Protein That Confers Broad-Spectrum White Rust Resistance in Arabidopsis thaliana to Four Physiological Races of Albugo candida. Molecular Plant-Microbe Interactions ${ }^{\circledR}$ 21(6): 757-768.

Borhan MH, Holub, EB, Epp, D, Kindrachuk, C, Omidi, M, Bozorgmanesh-Fard, G, Hegedus, D and Rimmer, SR. 2010. $W R R 4$, a broad spectrum TIR-NB-LRR gene from Arabidopsis thaliana that confers white rust resistance in transgenic oilseed brassica crops. Mol. Plant Pathol. 11:283-291.

Castel B, Fairhead S, Furzer OJ, Redkar A, Wang S, Cevik V, Holub EB, Jones JDG. 2021. Evolutionary trade-offs at the Arabidopsis $W R R 4 A$ resistance underpin alternate Albugo candida recognition specificities. Biorxiv. (simultaneous submission).

Cesari S, Thilliez G, Ribot C, Chalvon V, Michel C, Jauneau A, Rivas S, Alaux L, Kanzaki H, Okuyama Y, et al. 2013. The Rice Resistance Protein Pair RGA4/RGA5 Recognizes the Magnaporthe oryzae effectors AVR-Pia and AVR1-CO39 by Direct Binding. The Plant Cell 25(4): 1463.

705 Cevik V, Boutrot F, Apel W, Robert-Seilaniantz A, Furzer OJ, Redkar A, Castel B, Kover PX, Prince DC, Holub EB, et al. 2019. Transgressive segregation reveals mechanisms of Arabidopsis immunity to Brassica-infecting races of white rust Albugo candida. Proceedings of the National Academy of Sciences 116(7): 2767.

Choi YJ, Shin HD, Hong SB, Thines M. 2007. Morphological and molecular 710 discrimination among Albugo candida materials infecting Capsella bursapastoris world-wide. Fungal Diversity 27: 11 - 34.

Cooper AJ, Latunde-Dada AO, Woods-Tör A, Lynn J, Lucas JA, Crute IR, Holub EB. 2008. Basic Compatibility of Albugo candida in Arabidopsis thaliana and Brassica juncea Causes Broad-Spectrum Suppression of Innate Immunity. Molecular Plant-Microbe Interactions ${ }^{\circledR}$ 21(6): 745-756.

Dangl JL, Jones JDG. 2001. Plant pathogens and integrated defence responses to infection. Nature 411(6839): 826-833.

Dodds PN, Lawrence GJ, Catanzariti A-M, Teh T, Wang C-IA, Ayliffe MA, Kobe B, Ellis JG. 2006. Direct protein interaction underlies gene-for-gene specificity 720 and coevolution of the flax resistance genes and flax rust avirulence genes. Proceedings of the National Academy of Sciences 103(23): 8888.

Dou D, Kale SD, Wang X, Chen Y, Wang Q, Wang X, Jiang RH, Arredondo FD, Anderson RG, Thakur PB, et al. 2008. Conserved C-terminal motifs required for avirulence and suppression of cell death by Phytophthora sojae effector Avr1b. Plant Cell 20(4): 1118-1133.

Fairhead S. 2016. Translating genetics of oomycete resistance from Arabidopsis thaliana into Brassica production. PhD thesis, University of Warwick, UK. 
Furzer OJ, Cevik V, Fairhead S, Bailey K, Redkar A, Schudoma C, MacLean D, Holub EB, Jones JDG. 2021. PacBio Sequencing of the Albugo candida Ac2V genome reveals the expansion of the "CCG" class of effectors. Biorxiv. (simultaneous submission).

Geu-Flores F, Nour-Eldin HH, Nielsen MT, Halkier BA. 2007. USER Fusion: A rapid and efficient method for simultaneous fusion and cloning of multiple PCR products. Nucleic Acid Res 35(7): e55.

735 Guo H, Ahn HK, Sklenar J, Huang J, Ma Y, Ding P, Menke FLH, Jones JDG. 2020. Phosphorylation-Regulated Activation of the Arabidopsis RRS1-R/RPS4 Immune Receptor Complex Reveals Two Distinct Effector Recognition Mechanisms. Cell Host Microbe 27(5): 769-781.

Haas BJ, Kamoun S, Zody MC, Jiang RH, Handsaker RE, Cano LM, Grabherr M, Kodira CD, Raffaele S, Torto-Alalibo T, et al. 2009. Genome sequence and analysis of the Irish potato famine pathogen Phytophthora infestans. Nature 461(7262): 393-398.

Heller A, Thines M. 2009. Evidence for the importance of enzymatic digestion of epidermal walls during subepidermal sporulation and pustule opening in white blister rusts (Albuginaceae). Mycological Research 113(Pt 6-7): 657-667.

Holub EB, Brose E, Tör M, Clay C, Crute IR, Beynon JL. 1995. Phenotypic and genotypic variation in the interaction between Arabidopsis thaliana and Albugo candida. Molecular Plant Microbe Interact. 8(6):916-928.

Jia Y, McAdams SA, Bryan GT, Hershey HP, Valent B. 2000. Direct interaction of resistance gene and avirulence gene products confers rice blast resistance. Embo j 19(15): 4004-4014.

Jones JDG, Dangl JL. 2006. The plant immune system. Nature 444(7117): 323-329.

Jouet A, Saunders DGO, McMullan M, Ward B, Furzer O, Jupe F, Cevik V, Hein I, Thilliez GJA, Holub E, van Oosterhout C, Jones JDG. 2019. Albugo candida race diversity, ploidy and host-associated microbes revealed using DNA sequence capture on diseased plants in the field. New Phytologist 221(3): 1529-1543.

Jupe F, Witek K, Verweij W, Śliwka J, Pritchard L, Etherington GJ, Maclean D, Cock PJ, Leggett RM, Bryan GJ, et al. 2013. Resistance gene enrichment sequencing (RenSeq) enables reannotation of the NB-LRR gene family from sequenced plant genomes and rapid mapping of resistance loci in segregating populations. The Plant Journal 76(3): 530-544.

Kamoun S. 2006. A catalogue of the effector secretome of plant pathogenic oomycetes. Annu Rev Phytopathol 44: 41-60.

Kamoun S. 2007. Groovy times: filamentous pathogen effectors revealed. Curr Opin 765 Plant Biol 10(4): 358-365.

Kemen E, Gardiner A, Schultz-Larsen T, Kemen AC, Balmuth AL, RobertSeilaniantz A, Bailey K, Holub E, Studholme DJ, MacLean D, et al. 2011. Gene Gain and Loss during Evolution of Obligate Parasitism in the White Rust Pathogen of Arabidopsis thaliana. PLOS Biology 9(7): e1001094.

770 Livak KJ, Schmittgen TD. 2001. Analysis of Relative Gene Expression Data Using Real-Time Quantitative PCR and the 2- $\Delta \Delta$ CT Method. Methods 25(4): 402-408.

Ma S, Lapin D, Liu L, Sun Y, Song W, Zhang X, Logemann E, Yu D, Wang J, Jirschitzka J, et al. 2020. Direct pathogen-induced assembly of an NLR immune receptor complex to form a holoenzyme. Science 370: eabe3069.

775 Martin R, Qi T, Zhang H, Liu F, King M, Toth C, Staskawicz BJ. 2020. Structure of the activated Roq1 resistosome directly recognizing the pathogen effector XopQ. Science 370: eabd9993. 
McMullan M, Gardiner A, Bailey K, Kemen E, Ward BJ, Cevik V, RobertSeilaniantz A, Schultz-Larsen T, Balmuth A, Holub E, et al. 2015. Evidence for suppression of immunity as a driver for genomic introgressions and host range expansion in races of Albugo candida, a generalist parasite. eLife 4: e04550.

Meyers BC, Kozik A, Griego A, Kuang H, Michelmore RW. 2003. Genome-wide analysis of NBS-LRR-encoding genes in Arabidopsis. The Plant Cell 15(4): 809834.

785 Monteiro F, Nishimura MT. 2018. Structural, Functional, and Genomic Diversity of Plant NLR Proteins: An Evolved Resource for Rational Engineering of Plant Immunity. Annual Review of Phytopathology 56(1): 243-267.

Ngou BPM, Ahn H-K, Ding P, Jones JDG. 2021. Mutual potentiation of plant immunity by cell-surface and intracellular receptors. Nature. doi: 10.1038/s41586-021$790 \quad 03315-7$.

Oh SK, Young C, Lee M, Oliva R, Bozkurt TO, Cano LM, Win J, Bos JI, Liu HY, van Damme M, Morgan W, Choi D, Van der Vossen EA, Vleeshouwers VG, Kamoun S. 2009. In planta expression screens of Phytopthora infestans RXLR effectors reveal diverse phenotypes, including activation of the Solanum bulbocastanum disease resistance protein Rpi-blb2. Plant Cell 21: 2928-2947.

Pais M, Yoshida K, Giannakopoulou A, Pel MA, Cano LM, Oliva RF, Witek K, Lindqvist-Kreuze H, Vleehowers VG, Kamoun S. 2018. Gene expression polymorphism underpins evasion of host immunity in an asexual lineage of the Irish potato famine pathogen. BMC Evol Biol 18, 93.

800 Prince DC, Rallapalli G, Xu D, Schoonbeek H-J, Çevik V, Asai S, Kemen E, CruzMireles N, Kemen A, Belhaj K, et al. 2017. Albugo-imposed changes to tryptophan-derived antimicrobial metabolite biosynthesis may contribute to suppression of non-host resistance to Phytophthora infestans in Arabidopsis thaliana. BMC Biology 15(1): 20.

805 Ravensdale M, Bernoux M, Ve T, Kobe B, Thrall PH, Ellis JG, Dodds PN. 2012. Intramolecular Interaction Influences Binding of the Flax L5 and L6 Resistance Proteins to their AvrL567 Ligands. PLOS Pathogens 8(11): e1003004.

Rehmany AP, Gordon A, Rose LE, Allen RL, Armstrong MR, Whisson SC, Kamoun S, Tyler BM, Birch PRJ, Beynon JL. 2005. Differential Recognition of Highly

810 Divergent Downy Mildew Avirulence Gene Alleles by RPP1 Resistance Genes from Two Arabidopsis Lines. The Plant Cell 17(6): 1839.

Sarris PF, Duxbury Z, Huh SU, Ma Y, Segonzac C, Sklenar J, Derbyshire P, Cevik V, Rallapalli G, Saucet SB, et al. 2015. A Plant Immune Receptor Detects Pathogen Effectors that Target WRKY Transcription Factors. Cell 161(5): 1089-

8151100.

Schreiber KJ, Bentham A, Williams SJ, Kobe B, Staskawicz BJ. 2016. Multiple Domain Associations within the Arabidopsis Immune Receptor RPP1 Regulate the Activation of Programmed Cell Death. PLOS Pathogens 12(7): e1005769.

Segretin ME, Pais M, Franceschetti M, Chaparro-Garcia A, Bos JI, Banfield MJ, Kamoun S. 2014. Single amino acid mutations in the potato immune receptor R3a expand response to Phytophthora effectors. Mol Plant Microbe Interact 27(7): 624-637.

Tomborski J, Krasileva KV. 2020. Evolution of Plant NLRs: From Natural History to Precise Modifications. Annual Review of Plant Biology 71(1): 355-378.

825 Toruño TY, Stergiopoulos I, Coaker G. 2016. Plant-Pathogen Effectors: Cellular Probes Interfering with Plant Defenses in Spatial and Temporal Manners. Annual Review of Phytopathology 54(1): 419-441. 
Van de Weyer A-L, Monteiro F, Furzer OJ, Nishimura MT, Cevik V, Witek K, Jones JDG, Dangl JL, Weigel D, Bemm F. 2019. A Species-Wide Inventory of NLR Genes and Alleles in Arabidopsis thaliana. Cell 178(5): 1260-1272.e1214.

van der Hoorn RAL, Kamoun S. 2008. From Guard to Decoy: A New Model for Perception of Plant Pathogen Effectors. The Plant Cell 20(8): 2009.

Vleeshouwers VG, Raffaele S, Vossen JH, Champouret N, Oliva R, Segretin ME, Rietman H, Cano LM, Lokossou A, Kessel G, et al. 2011. Understanding and exploiting late blight resistance in the age of effectors. Annu Rev Phytopathol 49: 507-531.

Vleeshouwers VG, Rietman H, Krenek P, Champouret N, Young C, Oh SK, Wang M, Bouwmeester K, Vosman B, Visser RG, et al. 2008. Effector genomics accelerates discovery and functional profiling of potato disease resistance and phytophthora infestans avirulence genes. PLoS One 3(8): e2875.

Voglmayr H \& Riethmüller A. 2006. Phylogenetic relationships of Albugo species (white blister rusts) based on LSU rDNA sequence and oospore data. Mycological Research 110: 75 - 85 .

Wawra S, Trusch F, Matena A, Apostolakis K, Linne U, Zhukov I, Stanek J, Koźmiński W, Davidson I, Secombes CJ, et al. 2017. The RxLR Motif of the Host Targeting Effector AVR3a of Phytophthora infestans Is Cleaved before Secretion. The Plant Cell 29(6): 1184.

Whisson SC, Boevink PC, Moleleki L, Avrova AO, Morales JG, Gilroy EM, Armstrong MR, Grouffaud S, van West P, Chapman S, et al. 2007. A translocation signal for delivery of oomycete effector proteins into host plant cells. Nature 450(7166): 115-118.

Win J, Kanneganti TD, Torto-Alalibo T, Kamoun S. 2006. Computational and comparative analyses of 150 full-length cDNA sequences from the oomycete plant pathogen Phytophthora infestans. Fungal Genet Biol 43(1): 20-33.

Win J, Morgan W, Bos J, Krasileva KV, Cano LM, Chaparro-Garcia A, Ammar R, Staskawicz BJ, Kamoun S. 2007. Adaptive evolution has targeted the C-terminal domain of the RXLR effectors of plant pathogenic oomycetes. Plant Cell 19(8): 2349-2369.

Zhang YM, Shao ZQ, Wang Q, Hang YY, Xue JY. Wang B. Chen JQ. 2016. Uncovering the dynamic evolution of nucleotide-binding site-leucine-rich repeat (NBS-LRR) genes in Brassicaceae. Journal of Integrative Plant Biology 58(2): $165-177$.

865 Figure Legends:

Figure 1. Resistant and susceptible phenotypes of $W R R 4 A^{\mathrm{Col}-0}$ A. thaliana following inoculation of adult leaves with two different isolates of $\boldsymbol{A}$. candida Race 4.

Arabidopsis Col-0 is resistant to Race 4 isolate AcEm2 (collected from Capsella bursapastoris) and susceptible to Race 4 isolate AcEx1 (collected from A. halleri) whereas Ws-

8702 is susceptible to both isolates. Necrotic resistance observed with the Col-0-wrr4-6 mutant indicates that WRR $4 A$ is responsible for full green resistance to AcEm2. Fiveweek old plants were spray-inoculated with $A$. candida and incubated at $21{ }^{\circ} \mathrm{C}$; and then 
phenotyped 14 days after inoculation. Abaxial and adaxial picture of the same leaf are shown. Scale $=1 \mathrm{~cm}$.

875

Figure 2. Identification of eight CCG effectors from $A$. candida that elicit hypersensitive response when co-expressed in tobacco leaves with $W R R 4 A$ from Col-

\section{A. thaliana.}

(a) Pipeline for high-throughput screening of the CCG effectors for identification of recognized-CCGs against $W R R 4 A^{\mathrm{Col}-0}$ and $W R R 4 B^{\mathrm{Col}-0}$. The selected candidate effectors without the signal peptide (SP) were cloned into a USER expression vector under CaMV35S promoter for Agrobacterium-mediated transient expression either in N. tabacum or $N$. benthamiana. Recognized CCGs were identified by an HR readout and were further validated by a luciferase eclipse assay.

885 (b) The selection of candidate CCG effectors for screening against $W R R 4 A$ was based on positive selection, presence across different $A$. candida races and/or showing the characteristic of either allelic truncation or absence in a Col-virulent isolate of Race 4 (AcEx1). The recognized CCGs against WRR4A identified from our screen are highlighted with a yellow box and marked with an asterisk $(*)$.

890 (c) Transient expression of candidate CCG effectors either with GFP or with WRR $4 A^{\text {Col- }}$ 0 in $N$. tabacum. The eight identified CCGs trigger HR when co-expressed with $W R R 4 A^{\mathrm{Col}-0}$ but not with GFP alone. $W R R 4 A^{\mathrm{Col}-0}$ also do not show any auto-activity when co-delivered with the GFP control.

(d) The WRR4A-dependent CCG recognition correlates with the luciferase eclipse assay

895 upon particle bombardment in A. thaliana. Graph showing a drop in the luciferase activity of all recognized CCGs identified by a ratio of the measured luciferase in wildtype Col-0 as compared to that Col-wrr4a-6 mutant validating the recognition to be specific by $W R R 4 A^{\text {Col-0 }}$. Statistical significance versus Luciferase (Luc) alone ( $\mathrm{p}<0.05$, one way ANOVA, Bonferroni's multiple comparison test) is indicated by an asterisk. Error bars 900 indicate $\operatorname{SD}(\mathrm{n}=9)$.

Figure 3. The $W R R 4 B^{\text {Col-0 }}$ paralog recognizes four additional CCG effectors in $A$. candida.

(a) The selection of candidate CCG effectors for screening against $W R R 4 B^{\mathrm{Col}-0}$ based on their presence across isolates of different $A$. candida races. The recognized CCGs against $W R R 4 B^{\mathrm{Col}-0}$ identified from our screen are marked with an asterisk (*). 
(b) The luciferase eclipse assay upon particle bombardment in A. thaliana. Graph showing a drop in the luciferase activity of all recognized CCGs identified by a ratio of the measured luciferase in wildtype Col-0 as compared to a Col-wrr $4 b$ mutant validating the recognition to be specific by $W R R 4 B^{\mathrm{Col}-0}$. Statistical significance versus Luciferase

910 (Luc) alone ( $p<0.05$, one way ANOVA, Bonferroni's multiple comparison test) is indicated by an asterisk. Error bars indicate SD $(n=9)$.

(c) Multiple sequence alignment of $W R R 4 B$-recognized CCGs which shows homology primarily in the CCG motif. CCG motif is highlighted by red box.

Figure 4. The N-Terminal Portion of CCG effectors is sufficient for recognition by $915 W R R 4 A^{\mathrm{Col}-0}$ and occurs independent of the CCG motif

(a) Transient expression of different truncated versions of CCG28 either with GFP or with $W R R 4 A^{\text {Col-0 }}$ in N. tabacum. The N-terminal region that corresponds to aa 28-130 is mainly indispensable for $W R R 4 A^{\mathrm{Col}-0}$ recognition.

(b) Schematic representation of all the truncations infiltrated in Fig. 4a, showing either a

920 HR or a No-HR phenotype.

(c) The alignment of protein sequences of $\mathrm{N}$-terminal region of CCGs after signal peptide cleavage, up to amino acid 130 in all $W R R 4 A^{\mathrm{Col}-0}$ recognized CCGs. CCG motif is highlighted by red box.

(d) Mutations in CCG motif with CCG changed to AAG, CAA, CAG and AAA do not

925 impair $W R R 4 A^{\mathrm{Col}-0}$ recognition and HR. N. tabacum leaf was infiltrated to express wildtype or mutated variants of CCG motif in CCG28 a28-130 - YFP. All versions with wildtype as well as mutated CCG motif trigger HR, which was assessed at 48 hpi. Photographs are representative of three consistent replicates.

Figure 5. Differential recognition of CCG28 alleles across different $\boldsymbol{A}$. candida races.

930 (a) Schematic representation of the amino acid sequence alignment of CCG28 alleles from A. candida Race 2 (Ac2V), Race7 (Ac7v) and Race 4 (AcNc2, AcEm2 and AcEx1). The black region indicates conserved amino acid sequence across all races, whereas the grey region indicates variation. White bars in the alleles indicate the polymorphic sites where there is no predominant residue, and grey bars indicate polymorphic sites where

935 there is a predominant residue. The numbers next to the alleles refer to the total protein length. Figure drawn to scale. The CCG motif is indicated in green with a C.

(b) Quantitative recognition of different CCG28 alleles in N. tabacum transient assay. The different CCG28 alleles were co-infiltrated with $W R R 4 A^{\mathrm{Col}-0}$ into N. tabacum leaves. 
CCG28 shows recognition with an enhanced HR at 36 hpi. However, the AcEx1 CCG28

940 allele with a premature stop codon shows a delayed HR at 48 hpi, consistent with weak recognition of the CCG28 allele in an A. candida Race 4 isolate that overcomes the $W R R 4 A^{\mathrm{Col}-0}$ recognition.

(c) Expression analysis of CCG28 (relative to A. candida EF-1a) in Race 2 (Ac2v) and Race 4 (AcEm2 and AcEx1), showed that the AcEx1 truncated allele of CCG28 is weakly 945 expressed, likely contributing to virulence on $W R R 4 A^{\mathrm{Col}-0}$. Different letters indicate statistically significant differences between the different alleles tested (2 way ANOVA, Bonferroni's multiple comparison test, $\mathrm{p}<0.05$ ). Error bars represent SD. 